\title{
Knowledge and practices for pre-eclampsia and eclampsia care in Bangladesh
}

Kanij Sultana

Population Council

Amy Dempsey

Population Council

Follow this and additional works at: https://knowledgecommons.popcouncil.org/departments_sbsr-rh

Part of the Demography, Population, and Ecology Commons, Family, Life Course, and Society Commons, International Public Health Commons, and the Maternal and Child Health Commons How does access to this work benefit you? Let us know!

\section{Recommended Citation}

Sultana, Kanij and Amy Dempsey. 2017. "Knowledge and practices for pre-eclampsia and eclampsia care in Bangladesh," Ending Eclampsia Brief. Washington, DC: Population Council. 

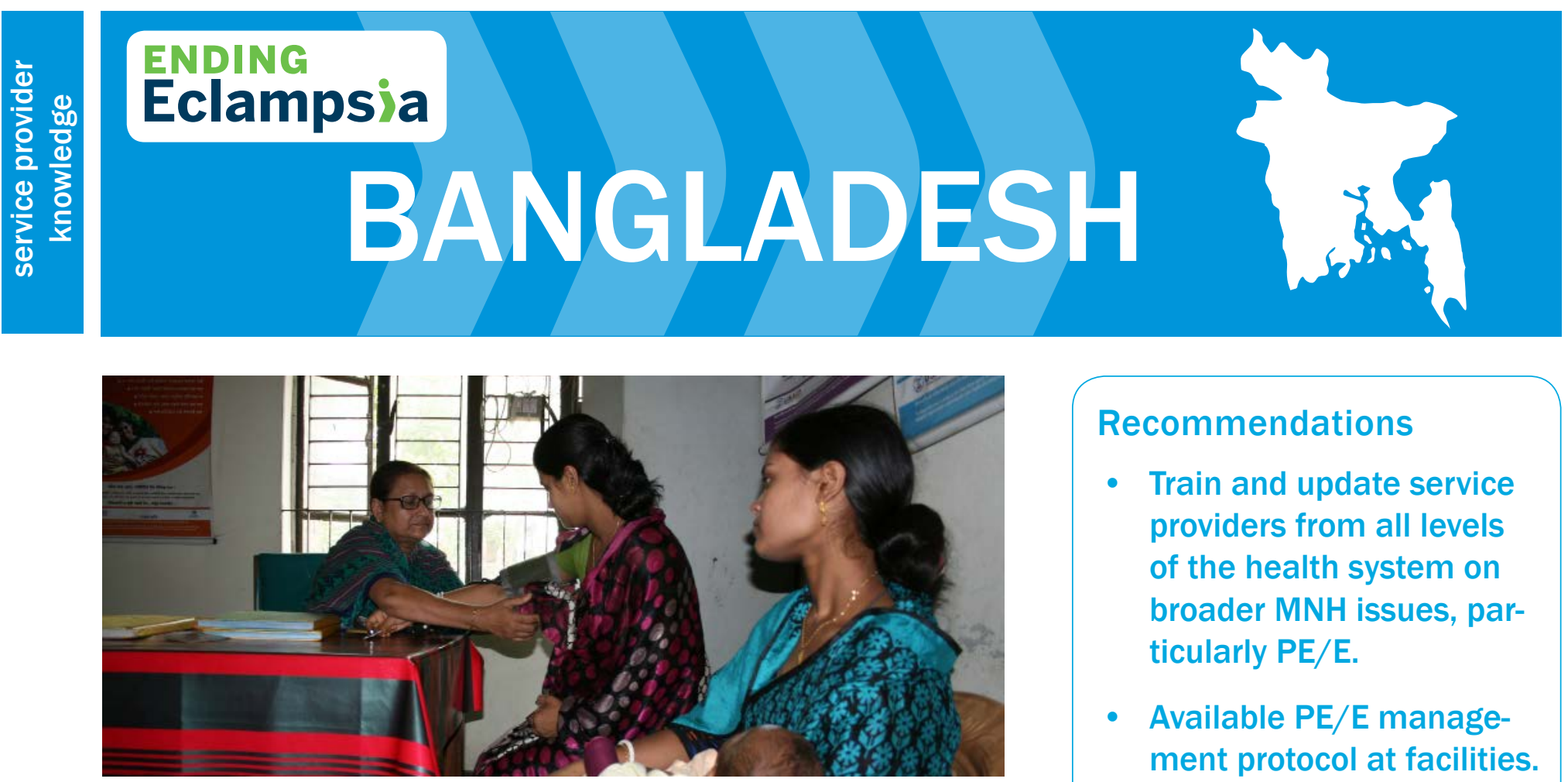

\section{KNOWLEDGE AND PRACTICES FOR PRE-ECLAMPSIA AND ECLAMPSIA CARE}

Pre-eclampsia is a leading cause of maternal and newborn morbidity and mortality worldwide. In Bangladesh, it is the second most common direct cause of maternal deaths. The Bangladesh Maternal Mortality Survey revealed that 20 percent - between 1,000 and 1,200 maternal deaths - are from pre-eclampsia and eclampsia (PE/E) each year.

Early detection and treatment during antenatal care (ANC) visits are instrumental in reducing deaths from $\mathrm{PE} / \mathrm{E}$. In Bangladesh, however, primary health care $(\mathrm{PHC})$ providers have limited knowledge of the condition, and in many cases are unaware of how to detect, manage, and treat it. This knowledge gap contributes to the high prevalence of $\mathrm{PE} / \mathrm{E}$-related deaths.

\section{RESEARCH}

This research brief presents the findings of a landscape analysis that was conducted to better understand service providers' maternal health knowledge, attitudes, and practices, particularly around PE/E.

Population Council researchers, in four districts and 12 upazilas, interviewed 289 service providers from 134 facilities. Among these service providers 79 were doctors, including those working in the maternal and child health unit. Researchers interviewed 210 other providers, which included family welfare visitors (FWV), sub-assistant community medical officers (SACMO), nurses, and medical assistants.

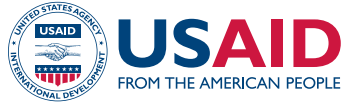

Made possible by the generous support of the American people through the United States

Agency for International Development (USAID).
The Population Council conducts research and delivers solutions that improve lives around the world. Big ideas supported by evidence: It's our model for global change. popcouncil.org (C) 2017 The Population Council, Inc

\section{Recommendations}

- Train and update service providers from all levels of the health system on broader MNH issues, particularly PE/E.

- Available PE/E management protocol at facilities.

- Organize competency based training for all PHC providers, with a refresher training after three months.

- Screen every patient in antenatal, delivery, and postnatal for increased blood pressure and assess for $\mathrm{PE} / \mathrm{E}$ risk.

- Review and revise curricula to include PE/E for nurses, midwives, FWVs, SACMOs and other paramedics.

- Providers should know of the antihypertensive and anticonvulsant drugs.

- A checklist can be used in facilities to assess PE/E risk and initiate prevention using calcium and aspirin. 


\section{FINDINGS}

\section{Service provider knowledge on detecting hypertension}

Eighty-four percent of doctors and 79 percent of other providers can correctly define hypertension as systolic blood pressure of $\geq 140 \mathrm{mmHg}$ and/or diastolic blood pressure of $\geq 90 \mathrm{mmHg}$. Seventeen percent of doctors and 22 percent of service providers are confused about the definition of hypertension.

All service providers were requested to identify signs and symptoms of pre-eclampsia, severe pre-eclampsia, and eclampsia. Results show that doctors have a strong understanding and other service providers had reasonably good knowledge of the signs and symptoms of PE, severe PE and eclampsia, including upper abdominal pain, headache, and blurred vision. They also mentioned some less important signs and symptoms, such as weight gain and oedema.

\section{Knowledge and practice for PE prevention}

Researchers assessed service provider's knowledge of risk factors of pre-eclampsia, including history of hypertension, diabetes, extreme maternal age $(<20$ or $>35$ years). Only 1.87 percent of providers observed in primary and secondary facilities looked for pre-eclampsia risk factors during antenatal care visits.

Researchers investigated service provider knowledge on prophylactic drugs, such as calcium supplements or lowdose aspirin, and found only 6 percent of doctors and no other providers are aware of the use of aspirin supplements to prevent pre-eclampsia.

No providers mentioned calcium supplements as prophylaxis, most likely because the drug is already regularly given during ANC. About 20 percent of doctors and 3 percent of other providers mentioned other drugs as prophylaxis, or said that they did not know of any prophylactic drugs, indicating confusion about prevention measures.

\section{Knowledge and practices for managing PE/E}

Researchers asked about commonly used antihypertensive drugs for managing mild, moderate, and severe hypertension in pregnancy. They also questioned providers about the appropriate blood pressure levels for administration of antihypertensive drugs.

About 77 percent of doctors and 19 percent of other service providers mentioned methyldopa or nifedipine as the drugs of choice for managing mild to moderate hypertension. Only 9 percent of doctors, and no other providers, mentioned hydralazine to manage hypertension.
Knowledge, attitudes, and practices for use of $\mathrm{MgSO}_{4}$

Service providers were asked to demonstrate their knowledge of the loading and maintenance doses of $\mathrm{MgSO}_{4}$ according to the Pritchard Regimen. Findings suggest that 35 percent of doctors and 1 percent of other providers know the correct loading dose of $\mathrm{MgSO}_{4}$ to be administered, and 36 percent of doctors and 1 percent of other service providers know the correct maintenance doses.

Although rare, toxicity of $\mathrm{MgSO}_{4}$ may occur, in which case calcium gluconate is the antidote. All service providers were asked if they knew of any drug used to treat toxicity, and only 30 percent of doctors and less than 1 percent of other providers know calcium gluconate as the remedy.

\section{Provider use of $\mathrm{MgSO}_{4}$}

Researchers asked service providers about their current use of $\mathrm{MgSO}_{4}$, its supply and availability at facilities, alternative drugs used to control seizures, and barriers to $\mathrm{MgSO}_{4}$ use. Only 37 percent of doctors and 4 percent of other providers reported they currently use $\mathrm{MgSO}_{4}$. Thirty three percent of providers reported sufficient supply, 5 percent overall reported inadequate supply, and the remaining 62 percent of providers reported none.

About 71 percent of doctors and 35 percent of other service providers mentioned some barriers to administering the loading dose of $\mathrm{MgSO}_{4}$ to severe $\mathrm{PE} / \mathrm{E}$ patients at primary facilities. Those barriers included the lack of training, lack of knowledge of side effects, lack of equipment or supplies, need for doctor supervision, fear of community reaction if patients were to die after use of $\mathrm{MgSO}_{4}$ and lack of proper monitoring.

About 39 percent of doctors and 4 percent of other providers use alternative drugs - diazepam and phenytoin - for controlling convulsions. Among those who reported using alternative drugs, 58 percent of doctors and 63 percent of other providers use an appropriate, though less safe and effective, alternative like diazepam.

Providers must be given more autonomy if they are to reduce $\mathrm{PE} / \mathrm{E}$-related deaths. With stronger knowledge of detection, prevention and management using antihypertensive drugs and $\mathrm{MgSO}_{4}$, providers at all levels of the health system can save the lives of women and babies.

\section{FOR MORE INFORMATION}

Kanij Sultana at Ksultana@popcouncil.org, info@endingeclampsia.org

For more information, visit www.endingeclampsia.org.

Citation: Warren, Charlotte, Sharif Hossain, Rahat Ara Nur, Kanij Sultana, Karen Kirk, Amy Dempsey. 2015. "Landscape Report on Pre-eclampsia and Eclampsia in Bangladesh." Washington, DC: Population Council. 